\title{
Nouveau regard sur la maladie mentale
}

\author{
M.-F. Bacqué \\ (C) Springer-Verlag France 2010
}

Dans les années 1990, un schizophrène présentant un cancer du poumon était hospitalisé en pneumologie. Discuté au staff, en présence du psychiatre qui le connaissait bien et le suivait depuis des années, le malade (ou du moins son dossier) se voyait proposer un traitement « à l'arrachée » par le psychiatre devant la réticence de tous... Il y a 20 ans en effet, il était encore difficile d'accepter de prendre certains risques avec les malades mentaux. Ils angoissaient les équipes, décevaient les médecins qui s'engageaient auprès d'eux, puis les perdaient de vue. Les échanges, entre les médecins somaticiens et les spécialistes de la santé mentale, commençaient fort heureusement à se déverrouiller et permettaient ces prises en charge transversales, aujourd'hui banalisées.

Les maladies physiques qui assombrissent la santé des malades mentaux ont été pendant longtemps négligées par nos sociétés. Le dualisme psychésoma sévissait encore, laissant croire que lorsqu'on était « fou », le corps était protégé. L'énergie psychique, tout entière employée dans le délire ou les désordres du cerveau, n'avait cure du corps, délaissé à un pur fonctionnement organique. Combien parlaient de «balancement psychosomatique » à propos des fous, qui les exonéraient d'investigations plus approfondies de la santé de ces patients. Une véritable remédicalisation de la psychiatrie était nécessaire pour certains services, tandis que les tabous et parfois même certains mythes retombaient, laissant apparaître la triste réalité de la santé des malades mentaux.

L'enfermement, la difficulté de prendre soin de soi, l'ignorance des messages de prévention, la peur qu'il engendre, l'abandon social, toutes ces raisons rendent le malade psychiatrique à très grand risque de développer des pathologies qui resteront trop longtemps non traitées. Regardons du côté de nos centres de détention, dans lesquels tant de personnes présentant des troubles psychiatriques sont « hébergées ». Certes, elles ont commis un délit et parfois un crime, mais la plupart du temps, leurs difficultés à s'adapter à notre société les poussent à une déviance facilitée par leur psychopathologie. Des chiffres comme $50 \%$ sont avancés ( $25 \%$ de

\section{M.-F. Bacqué ( $\square)$}

Département de psychologie, université Louis-Pasteur,

12, rue Goethe, F-67000 Strasbourg, France

e-mail : mfbacque@club-internet.fr troubles psychotiques chez les détenus) et montrent, lorsqu'on les compare aux 50000 lits de psychiatrie fermés depuis 20 ans, que les services psychiatriques doivent trouver des alternatives (on pense au secteur psychiatrique), mais surtout intégrer le soin et la prévention des maladies somatiques à la prise en charge ambulatoire.

Du côté des agents de santé primaire (les médecins généralistes pour nos institutions sanitaires), l'information doit être diffusée, surtout parce que certains malades psychiatriques s'adonnent à différentes toxicomanies ou se réfugient dans la marginalité, ce qui brouille les messages de prévention. Enfin, en cancérologie, les tabous seront, grâce à ce numéro spécial, largement démontés, pour permettre des soins de qualité sans le détour par des stéréotypes qui ont fait long feu.

Ce numéro de Psycho-oncologie consacré aux risques oncologiques des personnes présentant des troubles mentaux est très complet, sans être exhaustif, puisqu'il traite des schizophrénies, des psychoses plus généralement, des troubles bipolaires, des troubles de la personnalité. Une très vaste revue de question permettra à tous les médecins de faire le point sur les antidépresseurs en oncologie, enfin la question des tumeurs négligées, souvent énigmatique apportera un éclairage à de nombreux soignants, témoins révoltés parfois par l'arrivée à l'hôpital d'un patient pour lequel les possibilités thérapeutiques sont largement dépassées.

On retiendra la discussion éthique apportée par Silla Consoli et Sarah Dauchy que nous remercions vivement pour leur coordination du numéro. Pourquoi en effet les malades psychiatriques devraient-ils subir une telle perte de chance ? Cette question résume bien les interrogations posées par les autres articles. Les études montrent les liens complexes entre la maladie mentale (schizophrénies, dépression chronique) et la genèse ou la comorbidité oncologique. La discussion sur les facteurs confondants (alcoolisme vs dépression) et les facteurs médiateurs (la dépression favorisant le tabagisme, lui-même augmentant les risques de cancer) est un point délicat de l'interprétation des résultats statistiques retrouvés dans de nombreuses méta-analyses. Il y a donc un risque accru de cancer lorsqu'on a une maladie mentale chronique, de plus celle-ci a un pronostic plus défavorable que chez les malades équilibrés d'un point de vue psychique. Par ailleurs, les malades psychiatriques ont un 
accès aux soins défavorisé par leur moindre information, leur peur d'être malades physiquement, leur difficulté à appréhender des états corporels qui peuvent être confondus avec des effets psychiques désagréables (confusion, dépression, déréalisation). Enfin, les malades psychiatriques suscitent des appréhensions du côté des personnels médicaux et soignants qui redoutent leurs passages à l'acte ou leur décompensation.

Notre objectif, ici, est bien de dédramatiser ces difficultés, mais aussi d'instiller une réflexion éthique du côté des plus faibles. Plus d'information chez les soignants, plus d'échanges entre les services psychiatriques et les services d'oncologie (quitte à faire venir les uns et les autres lors d'échanges entre services), une meilleure articulation entre les prescriptions, quitte à employer également plus de techniques psychothérapiques pour limiter les interactions médicamenteuses délétères.

Ainsi, sans usurper les compétences des uns et des autres, les oncologues seront mieux formés à la dimension psychologique de la maladie du cancer, les psychiatres hésiteront moins à confier leurs malades atteints de cancer, les généralistes et les spécialistes en ville discerneront mieux les symptômes somatiques chez les malades psychiatriques, enfin, la population générale sera plus à même de protéger et de soigner ses membres "différents dans leur tête ", mais tout aussi dignes d'être bien traités lorsqu'ils sont malades. 